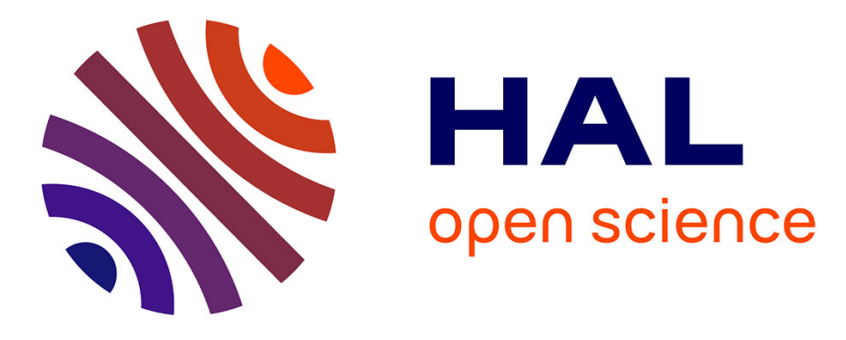

\title{
Magnetic Study of Co-Doped Magnetosome Chains
}

Lourdes Marcano, David Munoz, Rosa Martin-Rodriguez, Inaki Orue, Javier Alonso, Ana Garcia-Prieto, Aida Serrano, Sergio Valencia, Radu Abrudan, Luis Fernandez Barquin, et al.

\section{- To cite this version:}

Lourdes Marcano, David Munoz, Rosa Martin-Rodriguez, Inaki Orue, Javier Alonso, et al.. Magnetic Study of Co-Doped Magnetosome Chains. Journal of Physical Chemistry C, 2018, 122 (13), pp.75417550. 10.1021/acs.jpcc.8b01187 . hal-02976433

\section{HAL Id: hal-02976433 https://hal.science/hal-02976433}

Submitted on 23 Oct 2020

HAL is a multi-disciplinary open access archive for the deposit and dissemination of scientific research documents, whether they are published or not. The documents may come from teaching and research institutions in France or abroad, or from public or private research centers.
L'archive ouverte pluridisciplinaire HAL, est destinée au dépôt et à la diffusion de documents scientifiques de niveau recherche, publiés ou non, émanant des établissements d'enseignement et de recherche français ou étrangers, des laboratoires publics ou privés. 


\section{Magnetic Study of Co-Doped Magnetosome Chains}

Lourdes Marcano, ${ }^{\dagger}$ David Muñoz, ${ }^{\ddagger}$ Rosa Martín-Rodríguez, ${ }^{\S, \|}$ Iñaki Orue, ${ }^{\perp}$ Javier Alonso, ${ }^{\#}$

Ana García-Prieto, ${ }^{\nabla}$,\# Aida Serrano, ${ }^{\circ} \nabla^{\circledR}$ Sergio Valencia, ${ }^{\text {II }}$ Radu Abrudan, ${ }^{\text {Il }}$ Luis Fernández Barquín, ${ }^{+}$ Alfredo García-Arribas, ${ }^{\dagger, \#}$ Alicia Muela, ${ }^{\ddagger, \#}$ and M. Luisa Fdez-Gubieda*, ${ }^{*, \#[(])}$

${ }^{\dagger}$ Dpto. Electricidad y Electrónica, Universidad del País Vasco - UPV/EHU, 48940 Leioa, Spain

${ }^{\ddagger}$ Dpto. Inmunología, Microbiología y Parasitología, Universidad del País Vasco - UPV/EHU, 48940 Leioa, Spain

${ }^{\S}$ QUIPRE Department, University of Cantabria, Avda. de Los Castros 46, 39005 Santander, Spain

"Nanomedicine Group, IDIVAL, Avda. Cardenal Herrera Oria s/n, 39011 Santander, Spain

${ }^{\perp}$ SGIker, Universidad del País Vasco - UPV/EHU, 48940 Leioa, Spain

${ }^{\#}$ BCMaterials, Basque Center for Materials, Applications and Nanostructures, UPV/EHU Science Park, 48940 Leioa, Spain

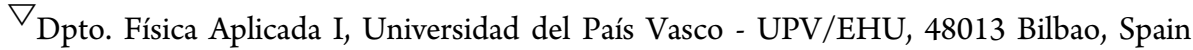

OSpLine, Spanish CRG BM25 Beamline, ESRF, 38000 Grenoble, France

Instituto de Ciencia de Materiales de Madrid, CSIC, Cantoblanco, 28049 Madrid, Spain

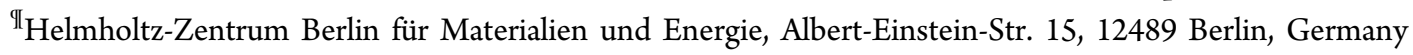

${ }^{+}$CITIMAC, Universidad de Cantabria, 39005 Santander, Spain

\section{Supporting Information}

ABSTRACT: Magnetotactic bacteria synthesize a chain of magnetic nanoparticles, called magnetosome chain, used to align and swim along the geomagnetic field lines. In particular, Magnetospirillum gryphiswaldense biomineralize magnetite, $\mathrm{Fe}_{3} \mathrm{O}_{4}$. Growing this species in a Co-supplemented medium, Co-doped magnetite is obtained, tailoring in this way the magnetic properties of the magnetosome chain. Combining structural and magnetic techniques such as transmission electron microscopy, energy-dispersive $\mathrm{x}$-ray spectroscopy, $\mathrm{X}$-ray absorption near edge structure, and $\mathrm{X}$-ray magnetic circular dichroism, we determine that $\sim 1 \%$ of $\mathrm{Co}^{2+}$ substitutes $\mathrm{Fe}^{2+}$ located in octahedral places in the magnetite, thus increasing the coercive field. In the framework

of the Stoner-Wohlfarth model, we have analyzed the evolution of the hysteresis loops as a function of temperature determining the different magnetic anisotropy contributions and their evolution with temperature. In contrast with the control magnetosome chains, whose effective anisotropy is uniaxial in the whole temperature range from 300 to $5 \mathrm{~K}$, the effective anisotropy of Codoped magnetosome chains changes appreciably with temperature, from uniaxial down to $150 \mathrm{~K}$, through biaxial down to $100 \mathrm{~K}$, to triaxial below $100 \mathrm{~K}$.

\section{INTRODUCTION}

Magnetotactic bacteria are microorganisms capable of aligning in and navigating along the geomagnetic field lines thanks to the chain of magnetic nanoparticles synthesized in their interior. Such a chain behaves like a compass needle in the presence of a magnetic field. ${ }^{1,2}$ Biomineralized magnetic nanoparticles, the so-called magnetosomes, have attracted a lot of scientific attention due to their exceptional properties compared with artificially synthesized counterparts, such as high chemical purity and crystallinity combined with the presence of a biocompatible lipidic membrane surrounding the magnetic nanoparticles. Hence these magnetosomes have been proposed for different nanotechnological applications, especially in the field of biomedicine. $^{3-8}$
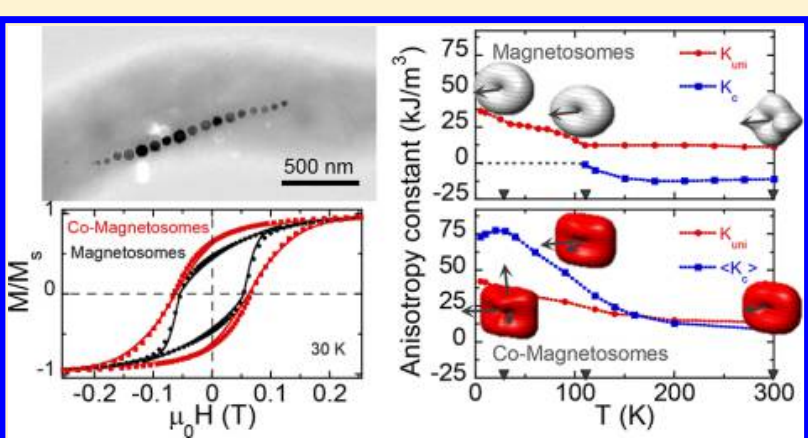

Published: March 15, 2018 depend on the species of mannetotic bacteria which introduces a high genetic control on their biomineralization. For example, Magnetospirillum gryphiswaldense MSR-1 produce magnetite cuboctahedral-shaped nanoparticles with an average size diameter of $\sim 45 \mathrm{~nm}$.

In the last years, several groups have proposed different strategies, focused on either in vivo processes ${ }^{9-13}$ or in vitro approaches, ${ }^{14-17}$ to tune the morphology, size, and composition of the magnetosomes to overcome the natural limitations

J. Phys. Chem 10.1021 /acs.jpcc.8b01187 
imposed by the genetic control of the magnetosome synthesis. One of the most promising approaches consists of modifying the composition of the magnetosomes by doping them with different elements. Magnetite, whose unit cell can be represented as $\mathrm{Fe}_{8}{ }^{3+}\left[\mathrm{Fe}^{2+} \mathrm{Fe}^{3+}\right]_{16} \mathrm{O}_{32}$, presents an inverse spinel structure and is essentially composed of $\mathrm{Fe}^{2+}$ ions in octahedral sites and $\mathrm{Fe}^{3+}$ ions split between octahedral and tetrahedral positions. The substitution of $\mathrm{Fe}^{2+}$ or $\mathrm{Fe}^{3+}$ cations by transitionmetal dopants in magnetite serves as a proven method to change the magnetic properties of the magnetic nanoparticles. ${ }^{18}$

Although reports addressing the possibility of doping magnetosomes with different metals date from the early $90 \mathrm{~s},{ }^{19,20}$ the first laboratory-controlled doping of magnetosomes in vivo was not performed until 2008 by Staniland et al. ${ }^{9}$ Since then, several groups have been able to dope magnetosomes with elements like $\mathrm{Mn}, \mathrm{Co}$, and $\mathrm{Cu}^{10-13}$ This opened up the possibility of controlled doping of magnetosomes under ambient conditions with a range of transition metals with the aim of obtaining magnetite-based magnetic nanoparticles with tunable properties for a wider range of applications. To this respect, doping the magnetosomes with $\mathrm{Co}$ is especially attractive to increase the magnetic coercivity and hence the hardness of the magnetosomes while the magnetic moment remains almost unaltered. In general, cobalt ferrite nanoparticles have attracted interest due to their large anisotropy combined with other properties like high Curie temperatures and good insulation, and they have been proposed as promising candidates for biomedical applications such as magnetic resonance imaging (MRI) and magnetic hyperthermia. ${ }^{21,22}$ Recently, cobalt-doped magnetosomes have been also tested, with good results, in magnetic hyperthermia. ${ }^{13}$

We provide detailed experimental and theoretical findings concerning the role of Co incorporation in the magnetic properties of the magnetosome chain with special focus on the magnetic anisotropy and its evolution with temperature. With this aim, we make use of state-of-the-art structural and magnetic characterization techniques such as transmission electron microscopy (TEM) with energy-dispersive X-ray spectroscopy (EDS), X-ray absorption near-edge structure (XANES), and Xray magnetic circular dichroism (XMCD). These techniques evidence the incorporation of small amounts of $\mathrm{Co}^{2+}(\sim 1 \%)$ in substitution of $\mathrm{Fe}^{2+}$ located in octahedral sites. DC magnetic measurements reveal that the incorporation of $\mathrm{Co}^{2+}$ introduces important changes in the coercive field of the hysteresis loop. Modeling of hysteresis loops with a modified StonerWohlfarth approach sheds light on the different anisotropy contributions and their dependence on temperature for magnetosomes and Co-doped magnetosome chains. At the same time, the evolution of the easy axis of magnetization is determined as well.

Besides the basic interest of the study, a good knowledge of the changes introduced by the $\mathrm{Co}$ in the magnetism of the magnetosome chain is decisive for future biomedical applications.

\section{EXPERIMENTAL SECTION}

Bacterial Strain and Growth Conditions. Magnetospirillum gryphiswaldense strain MSR-1 (DMSZ 6631) was employed in this work. The strain was cultured in a flask standard medium as described by Heyen and Schüler. ${ }^{23}$ The medium was enriched with either iron or both iron and cobalt just by adding either $100 \mu \mathrm{M}$ of $\mathrm{Fe}$ (III)-citrate (control magnetosomes) or $10 \mu \mathrm{M}$ of $\mathrm{Fe}(\mathrm{III})$-citrate and $100 \mu \mathrm{M}$ of
Co(II)-citrate (Co-magnetosomes) to the growth medium. Cultures were carried out in three-fourths $1 \mathrm{~L}$ bottles at $28{ }^{\circ} \mathrm{C}$ without shaking for $120 \mathrm{~h}$, when well-formed magnetosomes are observed.

Two different types of samples were prepared. First, for TEM-EDS and magnetic measurements the whole cells were employed. The final cultures were collected and fixed in $2 \%$ glutaraldehyde. Afterward, the cells were harvested by centrifugation, washed three times in $\mathrm{mQ}$ water, and concentrated up to $10^{8} \mathrm{cell} / \mathrm{mL}$.

Complementary XANES and XMCD measurements were carried out using extracted magnetosomes to increase the signal-to-noise ratio. Magnetosomes were isolated according to the protocol described by Grünberg et al. with minor modifications. $^{24}$ Cells were collected by centrifugation, suspended in $20 \mathrm{mM}$ HEPES-4 mM EDTA ( $\mathrm{pH} 7.4$ ), and disrupted using a French press $(1.4 \mathrm{kbar})$. The lysated cells were sonicated to promote the separation of magnetosomes and centrifuged at $600 \mathrm{~g}$ for $5 \mathrm{~min}$ to remove cell debris. Then, magnetosomes were collected from the supernatant by magnetic separation and rinsed 10 times with $10 \mathrm{mM}$ HEPES-200 mM NaCl (pH 7.4). Finally, the isolated magnetosomes were redispersed in ultrapure water.

Transmission Electron Microscopy and Energy-Dispersive X-ray Spectroscopy. Electron microscopy was performed on unstained cells adsorbed onto 300 mesh carbon-coated copper grids. TEM images were obtained with a JEOL JEM-1400 Plus electron microscope at an accelerating voltage of $120 \mathrm{kV}$. The particle size distribution was analyzed using a standard software for digital electron microscope image processing, ImageJ. ${ }^{25}$ Similar samples were employed for the EDS analysis, which was carried out in an FEI Tecnai F30 electron microscope at an accelerating voltage of $200 \mathrm{kV}$.

Magnetic Measurements. Magnetic characterization was carried out on the whole cells obtained as described before. The samples were freeze-dried and encapsulated in gelatin capsules. Isothermal magnetization loops were measured at magnetic fields between 4 and $-4 \mathrm{~T}$ at different temperatures, with no applied magnetic field in the cooling process on a cryogen-free vibrating sample magnetometer from Cryogenic. The zerofield-cooling/field-cooling ( $\mathrm{ZFC} / \mathrm{FC}$ ) magnetization curves were measured in a superconducting quantum interference device magnetometer (Quantum Design MPMS-3): The sample was cooled in the absence of any external field from $300 \mathrm{~K}$ to $5 \mathrm{~K}$. At $5 \mathrm{~K}$ a fixed magnetic field of $5 \mathrm{mT}$ was applied and the magnetization was measured upon warming to $300 \mathrm{~K}$ (ZFC). With the field still on, the sample was cooled to $5 \mathrm{~K}$ and the magnetization was measured upon warming to $300 \mathrm{~K}$ (FC).

X-ray Absorption Near-Edge Structure. XANES measurements were performed on isolated magnetosomes. For these measurements, extracted magnetosomes were freeze-dried and thoroughly mixed with boron nitride; the resultant mixture was compacted into $5 \mathrm{~mm}$ diameter pills.

The experiment was performed at room temperature and atmospheric conditions at the branch A of BM25-Spline of the ESRF synchrotron facility (France). ${ }^{26}$ The monochromator used in the experiments was a double crystal of $\mathrm{Si}(111)$. Fe and Co foils were measured at the beginning and end of the experiment for energy calibration. Under these conditions, the edge position of the sample can be determined with an accuracy of $0.3 \mathrm{eV}$. Measurements of $\mathrm{Fe}$ and $\mathrm{Co}$ foils, control magnetosomes $\left(\mathrm{Fe}_{3} \mathrm{O}_{4}\right)$, and a commercial reference of $\mathrm{CoFe}_{2} \mathrm{O}_{4}$ (purchased from Sigma-Aldrich) were measured in 


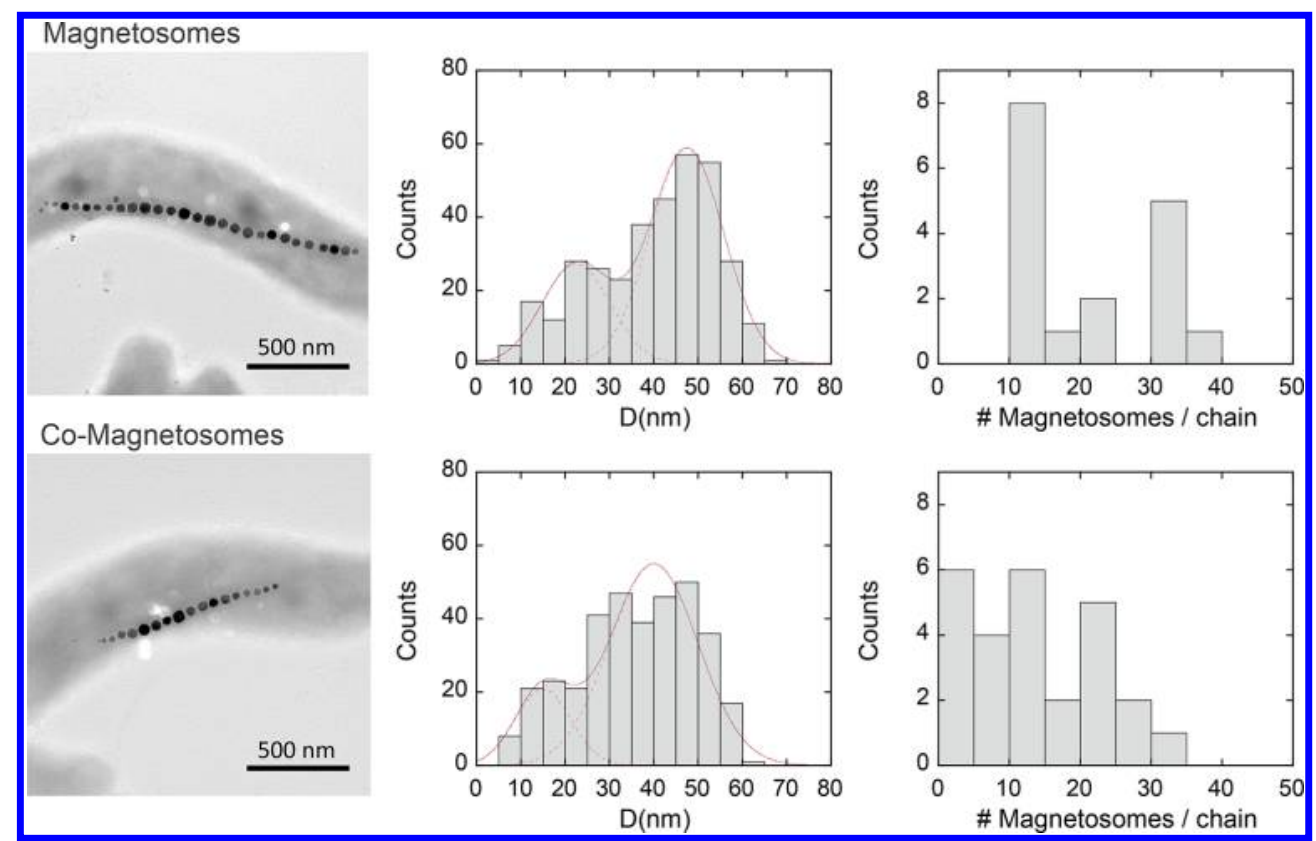

Figure 1. Representative TEM images, size distribution, and number of magnetosomes per chain for the control (top panel) and Co-doped magnetosomes (bottom panel).
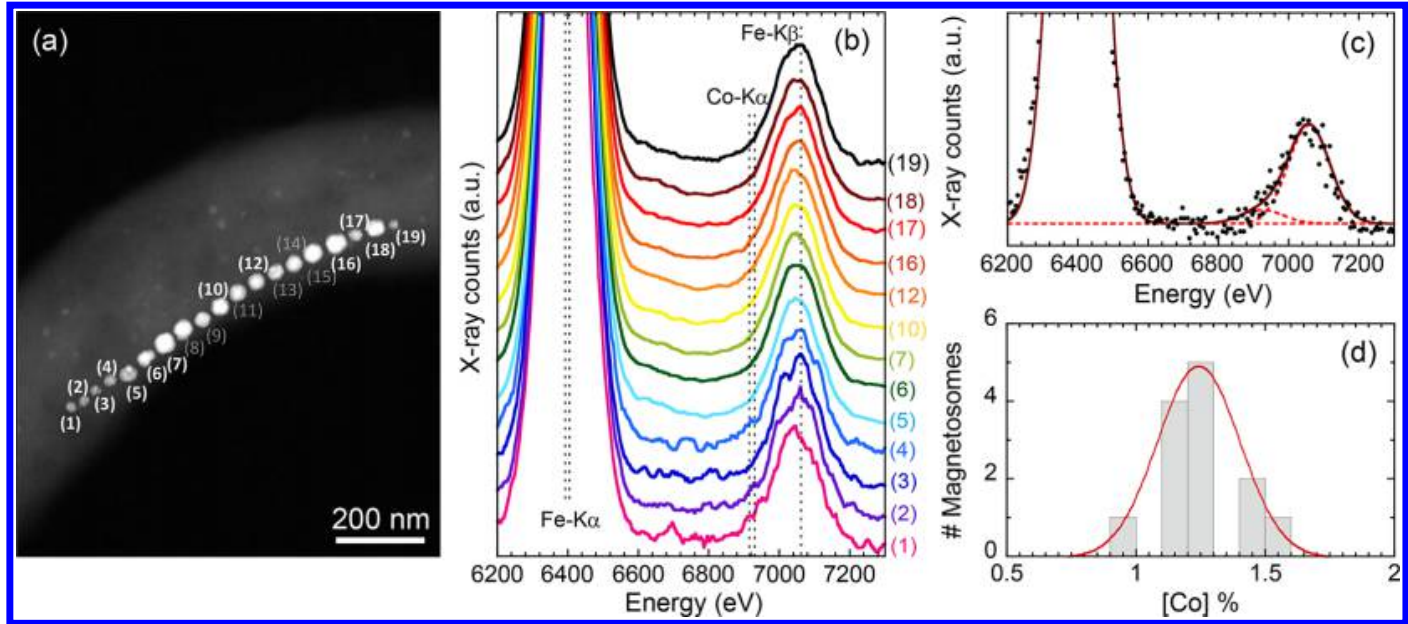

Figure 2. EDS measurements performed on individual magnetosomes inside a bacterium grown in a Co-supplemented medium. (a) TEM image of the analyzed magnetosomes chain within the cell. (b) EDS spectra of the 13 individual nanoparticles that have been analyzed. Dashed lines mark the position of the X-ray emission lines of Fe and Co. (c) Example of the fit to three Gaussian functions. (d) Distribution of the atomic \% Co fitted with a Gaussian function.

transmission mode at the $\mathrm{Fe}(7112 \mathrm{eV})$ and $\mathrm{Co}(7709 \mathrm{eV}) \mathrm{K}$ edge, respectively. Co-doped magnetosome sample was acquired in transmission mode at the $\mathrm{Fe} K$-edge and in fluorescence yield mode at the Co K-edge. From three to five spectra were acquired for each sample and merged to improve the signal-to-noise ratio. All of the data were treated by the Athena software from the Iffefit package. ${ }^{27}$

X-ray Magnetic Circular Dichroism. Room-temperature XMCD experiments were performed using the ALICE station $^{28,29}$ at the PM3 beamline of BESSY II in Berlin, Germany. A drop of $5 \mu \mathrm{L}$ of purified magnetosomes in aqueous solution $\left(20 \mu \mathrm{g} / \mathrm{mL}\right.$ of $\left.\mathrm{Fe}_{3} \mathrm{O}_{4}\right)$ was deposited onto silicon substrates. Data acquisition was done in total electron yield (TEY) mode with the incoming circularly polarized (right helicity) X-rays impinging at normal incidence with respect to the sample surface. A magnetic field of $\pm 0.27 \mathrm{~T}$ was applied along the beam propagation direction. X-ray absorption spectra (I) were obtained across the Fe $L_{2,3}$ with a step size of $0.2 \mathrm{eV}$. At each photon energy, data were acquired at magnetic remanence after positive $\left(I^{+}\right)$and negative $\left(I^{-}\right)$magnetic fields to yield the XMCD signal given by $I^{+}-I^{-}$.

\section{RESULTS AND DISCUSSION}

Structural Characterization. Figure 1 displays representative TEM images, size distribution, and number of magnetosomes per chain of control bacteria (top panel) and bacteria grown in a Co-supplemented medium (bottom panel). Control bacteria present uniformly sized magnetosomes except for those located at both ends of the chains, which are slightly smaller. This double size distribution is reflected in the histogram where one of the distributions is centered at $\langle D\rangle=$ $47 \mathrm{~nm}$ with a standard deviation $\sigma=8 \mathrm{~nm}$, with a majority of 


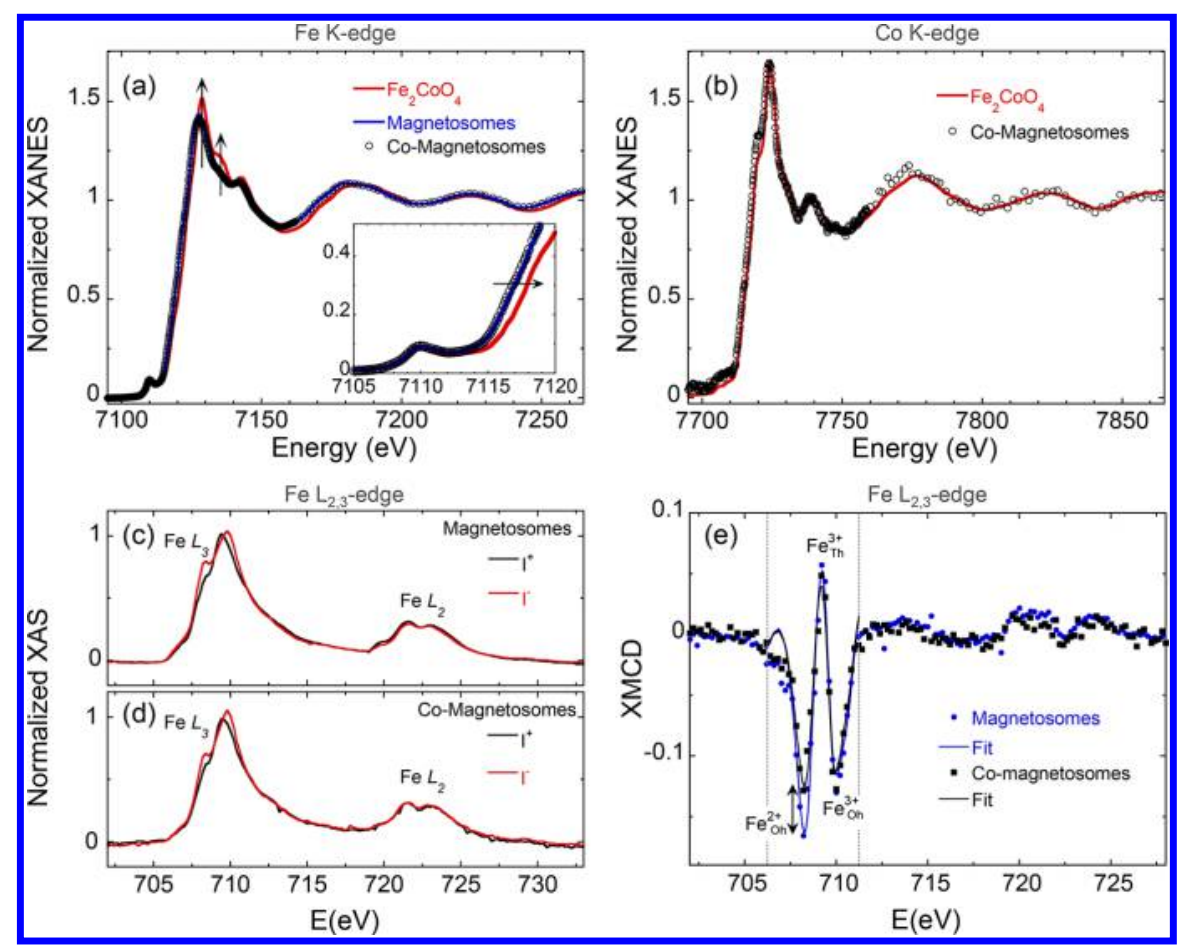

Figure 3. XANES spectra comparison (a) at $\mathrm{Fe} K$-edge of control magnetosomes (pure magnetite), cobalt ferrite $\left(\mathrm{CoFe}_{2} \mathrm{O}_{4}\right)$, and $\mathrm{Co}-\mathrm{doped}$ magnetosomes and (b) at $\mathrm{Co} K$-edge of $\mathrm{CoFe}_{2} \mathrm{O}_{4}$ and Co-doped magnetosomes. The inset in panel a shows the pre-edge region. X-ray absorption spectra (XAS) at Fe $L_{2,3}$-edges of (c) control magnetosomes and (d) Co-doped magnetosomes acquired at magnetic remanence after positive $\left(I^{+}\right)$ and negative $\left(I^{-}\right)$magnetic fields. Spectra have been normalized by the peak intensity at the $L_{3}$-edge of the nonmagnetic contribution to the XAS, that is, $\left(I^{+}+I^{-}\right) / 2$. Magnetic contribution, $I^{+}-I^{-}$, gives the XMCD signal presented in panel e, where the best linear combination fits have been superimposed. A second-degree polynomial background was subtracted from the experimental XMCD curves to account for a preexisting timedependent background.

magnetosomes, while that corresponding to a lower fraction of magnetosomes is centered at $\langle D\rangle=22 \mathrm{~nm}(\sigma=8 \mathrm{~nm})$. The average of magnetosomes per cell rounds to 20. Co-doped magnetosomes do also present the double size-distribution, but it is slightly shifted to lower values compared with the control magnetosomes. In this case, the size distribution peaks in $\langle D\rangle=$ $40 \mathrm{~nm}(\sigma=10 \mathrm{~nm})$ and $\langle D\rangle=15 \mathrm{~nm}(\sigma=6 \mathrm{~nm})$, respectively, while the average magnetosomes per chain decreases to 13 . TEM images do not show any clear difference in the morphology of the magnetosomes between both samples.

To estimate quantitatively the amount of Co incorporated into the magnetosomes, we have carried out a chemical analysis on the individual magnetosomes by means of EDS in TEM mode. As shown in Figure 2a, 13 magnetosomes randomly selected out of the 19 forming the chain have been analyzed, and their corresponding EDS spectra are shown in Figure $2 b$. The spectra show two main peaks. The one at lower energies $(\sim 6400 \mathrm{eV})$ corresponds to the $\mathrm{Fe}-\mathrm{K}_{\alpha}$ emission line, and the one at higher energies $(\sim 7060 \mathrm{eV})$ corresponds to the $\mathrm{Fe}-\mathrm{K}_{\beta}$. The $\mathrm{Co}-\mathrm{K}_{\alpha}$ emission line (at $\sim 6920 \mathrm{eV}$ ) introduces in the spectra a slight asymmetry in the low-energy region close to $\mathrm{Fe}-\mathrm{K}_{\beta}$ peak. We have fitted the spectra to three Gaussian functions centered at the positions of the $\mathrm{Fe}-\mathrm{K}_{\alpha}, \mathrm{Fe}-\mathrm{K}_{\beta}$, and $\mathrm{Co}-\mathrm{K}_{\alpha}$ emission lines (see Figure $2 \mathrm{c}$ ) and estimated the atomic percent of $\mathrm{Co}$ in the magnetosomes from the ratio of the integrated areas of the $\mathrm{Fe}-\mathrm{K}_{\alpha}$ and $\mathrm{Co}-\mathrm{K}_{\alpha}$ peaks. The analysis reveals that the atomic \% Co is small, ranging between 0.9 and $1.5 \%$ from one particle to another and that the Co content varies from particle to particle following a Gaussian distribution (Figure 2d). Even though the Co incorporation is small, it drastically changes the magnetic response, as will be shown later. Unlike previous results on $\mathrm{AMB}-1,{ }^{12}$ we have not found any relationships between atomic \% Co and the magnetosome size or position of the particle in the chain.

The arrangement of the Co ions in the magnetite structure has been investigated by using XANES. XANES is a very powerful technique that allows us to accurately determine the site occupancy of the Co cations in the spinel structure of the magnetite. Note that magnetite is an inverse spinel where the Fe ions occupy three different sites: $8 \mathrm{Fe}^{2+}$ in octahedral sites $\left(O_{\mathrm{h}}\right), 8 \mathrm{Fe}^{3+}$ in tetrahedral $\left(O_{\mathrm{t}}\right)$ sites, and $8 \mathrm{Fe}^{3+}$ in octahedral $\left(O_{\mathrm{h}}\right)$ ones.

Figure 3a shows the Fe K-edge XANES spectra of the control and Co-doped magnetosomes. As previously reported, ${ }^{30}$ the control sample accurately follows the spectrum expected for a high-quality magnetite standard. Co-doped magnetosomes display a very similar spectrum, consistent with the low Co content previously estimated from EDS measurements $(\sim 1 \%)$, which leaves the local environment around the $\mathrm{Fe}$ atoms practically unperturbed. The comparison with the $\mathrm{Fe} K$-edge XANES spectrum of commercial $\mathrm{CoFe}_{2} \mathrm{O}_{4}$ shows that, apart from differences in the intensity above the edge, the main changes expected from the Co doping should appear at the edge energy (see the inset of Figure 3a). In fact, the position of the edge energy is very sensitive to the oxidation state of the absorbing atom. $\mathrm{CoFe}_{2} \mathrm{O}_{4}$ is an almost inverse spinel with an inversion degree of $>80 \%$, ${ }^{31}$ where the $\mathrm{Co}^{2+}$ substitutes the $\mathrm{Fe}^{2+}$ ions at octahedral sites. As a consequence, the oxidation state of the $\mathrm{Fe}$ ions in magnetite $\left(\mathrm{Fe}^{2+}\right.$ and $\left.\mathrm{Fe}^{3+}\right)$ is lower than that in $\mathrm{CoFe}_{2} \mathrm{O}_{4}$ (mostly $\mathrm{Fe}^{3+}$ ), explaining the shift up of the energy edge of $\mathrm{CoFe}_{2} \mathrm{O}_{4} \approx 1.7 \mathrm{eV}$ toward higher energies. In our case, a $1 \%$ content of Co would mean an energy edge shift 
of $0.02 \mathrm{eV}$, which is undetectable because it is below the energy resolution $(0.3 \mathrm{eV})$.

On the contrary, XANES on the Co K-edge probes the local environment of $\mathrm{Co}$ atoms in doped magnetosomes. As shown in Figure $3 \mathrm{~b}$, the spectrum is comparable to that of $\mathrm{CoFe}_{2} \mathrm{O}_{4}$. The edge position is the same in both cases, revealing that the oxidation state of the Co atoms in the Co doped magnetosomes is $\mathrm{Co}^{2+}$, and the absence of a pre-edge peak strongly suggests a predominant octahedral coordination for these $\mathrm{Co}^{2+}$ ions. $^{32}$

These results have been confirmed by XMCD experiments at the $\mathrm{Fe} L_{2,3}$-edges. Figure $3 \mathrm{c}, \mathrm{d}$ shows the normalized absorption spectra at the $\mathrm{Fe} L_{2,3}$-edges of the control and Co-doped magnetosomes. As shown in Figure $3 \mathrm{e}$, the resulting $L_{3} \mathrm{XMCD}$ spectrum of the control magnetosomes consists of three main components related to the three different iron occupations of magnetite. The sign of the magnetic dichroism for each component is defined by the direction of its magnetic moment. Because $\mathrm{Fe}^{2+}$ and $\mathrm{Fe}^{3+}$ in octahedral places are aligned ferromagnetically, negative intensities are obtained for both, while for $\mathrm{Fe}^{3+}$ placed in tetrahedral sites, coupled antiferromagnetically with the $\mathrm{Fe}^{3+}$ in octahedral sites, the peak shows a positive intensity. By comparing XMCD data with theoretical spectra for each individual component, ${ }^{33,34}$ the site occupancies of the $\mathrm{Fe}$ cations have been determined. The best linear combination fits obtained from the three theoretical components already mentioned between 706.0 and $711.3 \mathrm{eV}$ have been superimposed in Figure 3e. For the control magnetosomes, the fit results give a ratio of $\mathrm{Fe}_{\mathrm{Oh}}^{2+}: \mathrm{Fe}_{\mathrm{Th}}^{3+}: \mathrm{Fe}_{\mathrm{Oh}}^{3+}$ of $0.98(4): 1.00(5): 1.07(5)$, close to the expected ratio for stoichiometric magnetite $(1: 1: 1)$. In contrast, for Co-doped magnetosomes the fit gives a ratio of $0.85(4): 1.00(5): 1.19(6)$. The significant decrease of $4 \%$ in the $\mathrm{Fe}_{\mathrm{Oh}}^{2+}$ peak intensity, even evident at a first glance, indicates that the $\mathrm{Co}^{2+}$ ions are substituting the octahedral $\mathrm{Fe}^{2+}$ ions. The percentage of Co estimated by XMCD (4\%) is significantly higher than the one obtained from the EDS analysis (1\%). This discrepancy is attributed to the short probing depth $(3-5 \mathrm{~nm})$ of the XMCD measurements, performed in TEY mode, and suggests a higher cobalt concentration on the surface of magnetosomes than in the core.

Therefore, according to XANES and XMCD, the low Co content of the Co-doped magnetosomes barely alters the inverse spinel structure. The Co ions are mostly incorporated as $\mathrm{Co}^{2+}$ in octahedral sites by substituting $\mathrm{Fe}^{2+}$. These results agree with previous ones reported in the References section. ${ }^{9,12}$

Magnetic Measurements. The incorporation of Co into the magnetosome structure modifies the magnetic properties, and hereafter we will analyze those changes. As previously indicated, all of the measurements were carried out on the whole bacteria and hence on magnetosomes arranged in chains.

Figure 4 shows the zero-field cooling/field cooling (ZFC/ FC) curves of the control and Co-doped magnetosome chains. Control magnetosome chains present a marked irreversibility in the whole studied temperature range, with the blocking temperature being above $300 \mathrm{~K}$ as expected due to the large size of the magnetosomes. The ZFC curve displays a sharp transition at $T_{\mathrm{V}}=107 \mathrm{~K}$ corresponding to the well-known Verwey transition, a cubic-to-monoclinic crystallographic phase transition characteristic of magnetite. The Verwey transition occurs at lower temperatures in magnetosomes than in bulk magnetite $(\sim 120 \mathrm{~K})$, as previously observed. ${ }^{35-37}$ The fact that the Verwey transition is so abrupt reflects the good

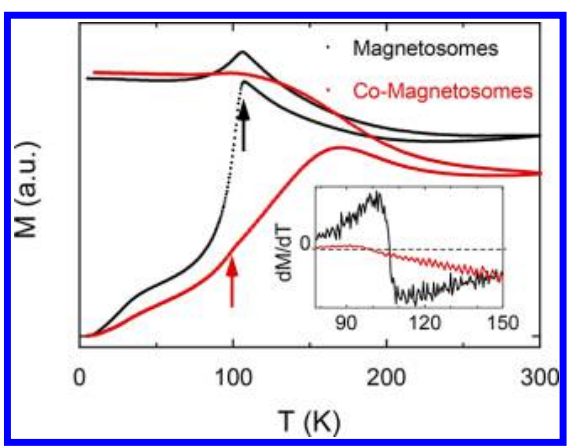

Figure 4. $\mathrm{ZFC} / \mathrm{FC}$ curves at $50 \mathrm{mT}$ of the control and Co-doped magnetosome chains. The inset displays the derivative of FC for both samples. Black and red arrows highlight the Verwey transition in control and Co-doped magnetosomes, respectively.

stoichiometry of the biomineralized magnetite. At $30 \mathrm{~K}$ the ZFC presents a shoulder attributed to the ordering of electron spins in magnetite at low temperature. ${ }^{38-40}$ The Verwey transition is also observed in the FC curve and in its derivative (see inset Figure 4), but in this case the magnetization decreases only slightly below $T_{\mathrm{V}}$ and remains constant down to $5 \mathrm{~K}$. In the FC curve the low-temperature transition is absent.

As in the control, the ZFC/FC curves of Co-doped magnetosome chains show clear irreversibility, but the shape of the curves is markedly different. In the ZFC curve a wide maximum stands out at $\sim 150 \mathrm{~K}$. The Verwey transition is only barely discerned at $100 \mathrm{~K}$, and the low-temperature transition is almost vanished. On the contrary, the FC magnetization increases monotonically as the temperature decreases until it reaches a plateau below the Verwey transition $(100 \mathrm{~K})$, identified as the point at which the derivative of the FC curve becomes null (see inset in Figure 4).

The $\mathrm{M}(\mathrm{H})$ loops of the control and Co-doped magnetosome chains performed at 300,110, and $30 \mathrm{~K}$ are shown in Figure $5 \mathrm{a}-\mathrm{c}$. At $300 \mathrm{~K}$ the $\mathrm{M}(\mathrm{H})$ loops overlap, but as the temperature

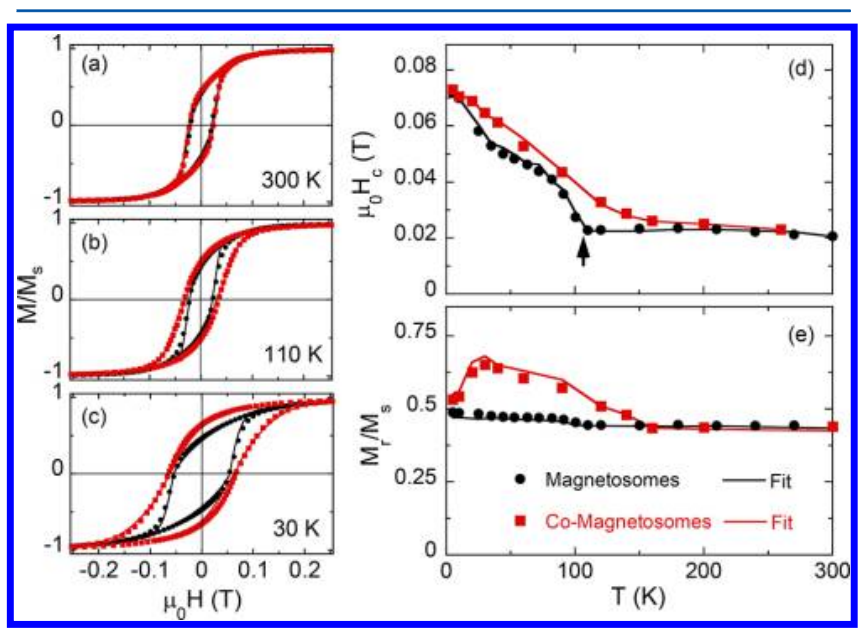

Figure 5. Hysteresis loops of the control and Co-doped magnetosome chains measured at (a) 300, (b) 110, and (c) $30 \mathrm{~K}$. Continuous lines correspond to the simulated loops considering the anisotropy constants shown in Figure 6. (d) Coercive field $\left(\mu_{0} H_{c}\right)$ and (e) reduced remanence magnetization $\left(M_{\mathrm{r}} / M_{\mathrm{s}}\right)$ comparison as a function of temperature. Continuous lines correspond to the values obtained from the simulation considering the anisotropy constants shown in Figure 6. The black arrow in panel d marks the Verwey transition $\left(T_{\mathrm{V}}\right)$ of the control magnetosomes. 


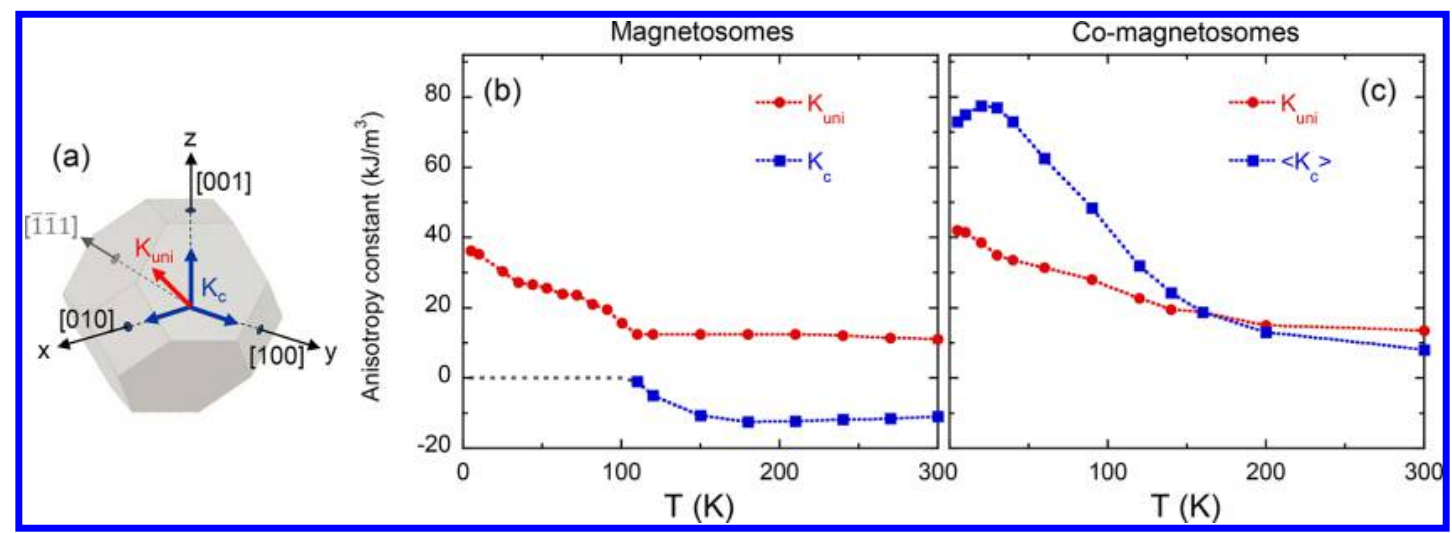

Figure 6. (a) Schematic representation of an individual magnetosome where $x, y$, and $z$ conform the coordinate axes selected in the simulations coincident to $\langle 100\rangle$ directions. Uniaxial axis (red direction) forms $25^{\circ}$ with the $\langle 111\rangle$ direction. Temperature evolution of the anisotropy constants for (b) control magnetosomes and (c) Co-doped magnetosomes. Red dots correspond to the uniaxial anisotropy constant $\left(K_{\text {uni }}\right)$ and blue ones represent the cubic magnetocrystalline anisotropy constant $\left(K_{c}\right)$.

decreases clear differences in the shape of the loops arise. These differences between the control and Co-doped magnetosome chains can be easily tracked in the plots of the coercive field $\left(\mu_{0} H_{\mathrm{c}}\right)$ and reduced remanence $\left(M_{\mathrm{r}} / M_{\mathrm{s}}\right)$ versus temperature shown in Figure 5d,e.

In the control magnetosome chains, the coercivity is nearly constant from $300 \mathrm{~K}$ down to $T_{\mathrm{V}}$ with $\mu_{0} H_{\mathrm{c}} \approx 22 \mathrm{mT}$. Below $T_{\mathrm{V}}$, the coercivity increases steeply up to $50 \mathrm{mT}$ at the lowtemperature transition occurring at $30 \mathrm{~K}$, after which the slope increases again to $73 \mathrm{mT}$ at $5 \mathrm{~K}$. Conversely, the $M_{\mathrm{r}} / M_{\mathrm{s}}$ value remains fairly constant between 0.44 and 0.49 throughout the whole temperature range. These values around 0.5 indicate that randomly oriented magnetosome chains behave like uniaxial magnetic domains in the framework of the Stoner-Wohlfarth model, ${ }^{41}$ as will be discussed in the following.

Despite the low Co content, the magnetic response of the Co-doped magnetosome chains changes significantly. Regarding $\mu_{0} H_{\mathcal{c}}$ instead of two well-defined kinks as those found in the control sample, here a progressive increase takes place as the temperature decreases, moderately down to $\sim 150 \mathrm{~K}$ and steeply after that point down to $5 \mathrm{~K}$. The coercive field at $300 \mathrm{~K}$ $(20 \mathrm{mT})$ is coincident with the value found for the control magnetosomes, but at lower temperatures it becomes consistently higher, up to $60 \%$ higher than the coercive field of the control sample. The reduced remanence is nearly constant from $300 \mathrm{~K}$ down to $150 \mathrm{~K}$ at $\sim 0.45$, similarly to the control magnetosomes, but increases notably up to $M_{\mathrm{r}} / M_{\mathrm{s}}=$ 0.65 at $30 \mathrm{~K}$ and drops steeply afterward, down to 0.53 at $5 \mathrm{~K}$.

Magnetic Model. To understand the observed features in the magnetic measurements caused by the $\mathrm{Co}^{2+}$ incorporation into the magnetosomes structure and, in particular, its role in the effective anisotropy, we have carried out numerical simulations of the magnetization dynamics of the magnetosomes at different temperatures by using an approach based on the Stoner-Wohlfarth model with some pertinent modifications. $^{42-44}$ All of the data analysis has been performed considering a unique magnetic phase.

In this framework, magnetosome chains have been considered as a collection of independent magnetic dipoles, where the equilibrium orientation of each magnetic dipole is calculated by minimizing the single dipole energy density $E$ that is calculated as the sum of three contributions: ${ }^{45-48}$ (i) the magnetocrystalline anisotropy energy $\left(E_{\mathrm{c}}\right)$; (ii) an effective uniaxial anisotropy term arising from the competition between the magnetosome shape anisotropy and the dipolar interactions between magnetosomes in the chain $\left(E_{\text {uni }}\right)$; and (iii) the Zeeman energy term $\left(E_{\mathrm{Z}}\right)$.

In spherical coordinates, considering the $\langle 100\rangle$ crystallographic directions as the reference system (Figure 6a), the first term of the single dipole energy density is the cubic magnetocrystalline energy given by

$$
E_{c}(\theta, \varphi)=K_{c}\left[\sin ^{4}(\theta) \sin ^{2}(\varphi)+\sin ^{2}(2 \theta) / 4\right]
$$

where $K_{\mathrm{c}}$ is the cubic anisotropy constant and $\theta$ and $\varphi$ are the polar and azimuthal angles, respectively, of the magnetic moment (whose direction is given by the unit vector $\hat{u}_{m}$ ).

The second term is an effective uniaxial anisotropy contribution along the $\hat{u}_{\text {uni }}$ axis that results from the competition of the shape anisotropy of the magnetosomes and the interparticle dipolar interactions between nearest neighbors in the chain

$$
E_{\text {uni }}(\theta, \varphi)=K_{\text {uni }}\left[1-\left(\hat{u}_{m} \cdot \hat{u}_{\text {uni }}\right)^{2}\right]
$$

where $K_{\text {uni }}$ is the uniaxial anisotropy constant. The justification for the representation of the interaction energy as a uniaxial anisotropy term lies in recent small-angle neutron scattering experiments, which reveal that the chain is composed of singledomain particles whose magnetic moments rotate coherently. ${ }^{48}$ The direction of the uniaxial axis $\hat{u}_{\text {uni }}$ would point along the chain axis if only the dipolar interactions were present. The chain axis is one of the magnetosome $\langle 111\rangle$ axes (see Figure 6a) because it is well known that magnetosomes arrange along the chain with their hexagonal faces toward each other. ${ }^{49,50}$ However, the shape anisotropy, an important contribution due to the strongly faceted structure of the magnetosomes, seems to point away from the chain axis, ${ }^{51}$ and, in fact, our simulations improve considerably when the uniaxial easy axis is tilted $25^{\circ}$ out of the chain axis toward the $\langle 110\rangle$ directions; see Figure $6 \mathrm{a}^{48}$

The third term is the Zeeman energy in an external magnetic field $\mu_{0} \vec{H}$

$$
E_{\mathrm{Z}}(\theta, \varphi)=-\mu_{0} M H\left(\hat{u}_{\mathrm{H}} \cdot \hat{u}_{m}\right)
$$

where $\hat{u}_{\mathrm{H}}$ represents the external magnetic field unit vector.

The single dipole energy density $E$ is thus given by

$$
E(\theta, \varphi)=E_{\mathrm{c}}+E_{\mathrm{uni}}+E_{\mathrm{Z}}
$$


The hysteresis loops have then been calculated following a dynamical approach in which the single-domain magnetization can switch between the available energy minima states, at a rate determined by a Boltzmann factor $\left(\exp \left(-\frac{V \Delta E}{k_{\mathrm{B}} T}\right)\right)$, where $\mathrm{V}$ is the particle volume and $\Delta E$ is the energy barrier between each pair of minima states, as well-explained in refs 42-44. In each simulation, $K_{\mathrm{c}}$ and $K_{\mathrm{uni}}$ have been adjusted at each temperature to achieve the best match between experiment and theory. As shown in Figure $5 \mathrm{a}-\mathrm{c}$, the proposed model accurately reproduces the experimental hysteresis loops and the thermal evolution of the coercivity and reduced remanence (Figure $5 \mathrm{~d}, \mathrm{e})$ for both the control and the Co-doped magnetosomes.

The thermal evolution obtained for $K_{\mathrm{c}}$ and $K_{\mathrm{uni}}$ for the control magnetosomes is shown in Figure $6 \mathrm{~b}$. From $300 \mathrm{~K}$ down to the Verwey temperature $T_{\mathrm{V}}$, the evolution of $K_{\mathrm{c}}$ reproduces the values and trend reported for bulk monocrystalline magnetite (at $\left.300 \mathrm{~K}, K_{\mathrm{c}}=-11 \mathrm{~kJ} / \mathrm{m}^{3}\right) .{ }^{52}$ On the contrary, $K_{\text {uni }}$ remains constant $\left(11\right.$ to $12 \mathrm{~kJ} / \mathrm{m}^{3}$ ) down to $T_{\mathrm{V}}$, as expected, because shape anisotropy and the strength of magnetic interactions depend only slightly on temperature in this range of temperatures. Below $T_{\mathrm{V}}$, the magnetocrystalline anisotropy changes from cubic to essentially uniaxial along the $\langle 100\rangle$ directions of the original cubic spinel structure. Therefore, below $T_{\mathrm{V}}$, the effective anisotropy is purely uniaxial (eq 2) and results from the competition between the magnetocrystalline uniaxial anisotropy and the shape and interaction contribution. The resulting uniaxial anisotropy constant $K_{\text {uni }}$ increases substantially from 11 to $12 \mathrm{~kJ} / \mathrm{m}^{3}$ at $T_{\mathrm{V}}$ to $37 \mathrm{~kJ} / \mathrm{m}^{3}$ at $5 \mathrm{~K}$, as previously observed. ${ }^{53}$

In the Co-doped magnetosomes, a large positive contribution to the cubic magnetocrystalline energy is observed as expected from previous works in Co-substituted magnetite. ${ }^{52,53}$ In these calculations, a Gaussian distribution of the cubic anisotropy constant $K_{\mathrm{c}}$ has been introduced to account for the EDS results, from which the atomic \% Co rounds $1 \%$ and varies from particle to particle following a Gaussian distribution (Figure $2 \mathrm{~d})$. The mean magnetocrystalline anisotropy constant $\left\langle K_{\mathrm{c}}\right\rangle$ changes substantially, appearing strongly dependent on temperature. Both the observed temperature dependence and the obtained anisotropy values of $K_{c}$ agree with the results found in the bibliography considering a concentration of $1 \%$ Co: ${ }^{52,54,55} K_{c}$ is positive in the entire temperature range, meaning that unlike in magnetite, the magnetocrystalline easy axes are the $\langle 100\rangle$, and $K_{c}$ increases considerably from a value of $8 \mathrm{~kJ} / \mathrm{m}^{3}$ at $300 \mathrm{~K}$ to $78 \mathrm{~kJ} / \mathrm{m}^{3}$ at $30 \mathrm{~K}$. On the contrary, $K_{\text {uni }}$ shows a similar trend as the one observed for the control magnetosomes. This is not surprising given the origin of this uniaxial anisotropy contribution because Co doping does not introduce any change in the magnetosome shape and arrangement in the chains, as revealed by TEM.

The implications of the anisotropy constant values in the effective easy axes are reflected in the zero-field energy surfaces plotted in Figure 7 (and Movies S1-S6) at selected temperatures. For the control magnetosomes, at $300 \mathrm{~K}$ (see Figure $7 \mathrm{a}$ ) the energy surface shows one single minimum, meaning that the effective anisotropy is uniaxial. The position of this minimum defines the direction of the corresponding easy axis, which in this case is $\left(\theta=74^{\circ}, \varphi=45^{\circ}\right)$. Thus even though the cubic magnetocrystalline contribution $\left(E_{\mathrm{c}}\right)$ corresponding to a negative $K_{c}(\langle 111\rangle$ easy axes $)$ is well distinguished in the shape of the energy surface at $300 \mathrm{~K}$, it definitely plays a minor role in the overall energy, and its main

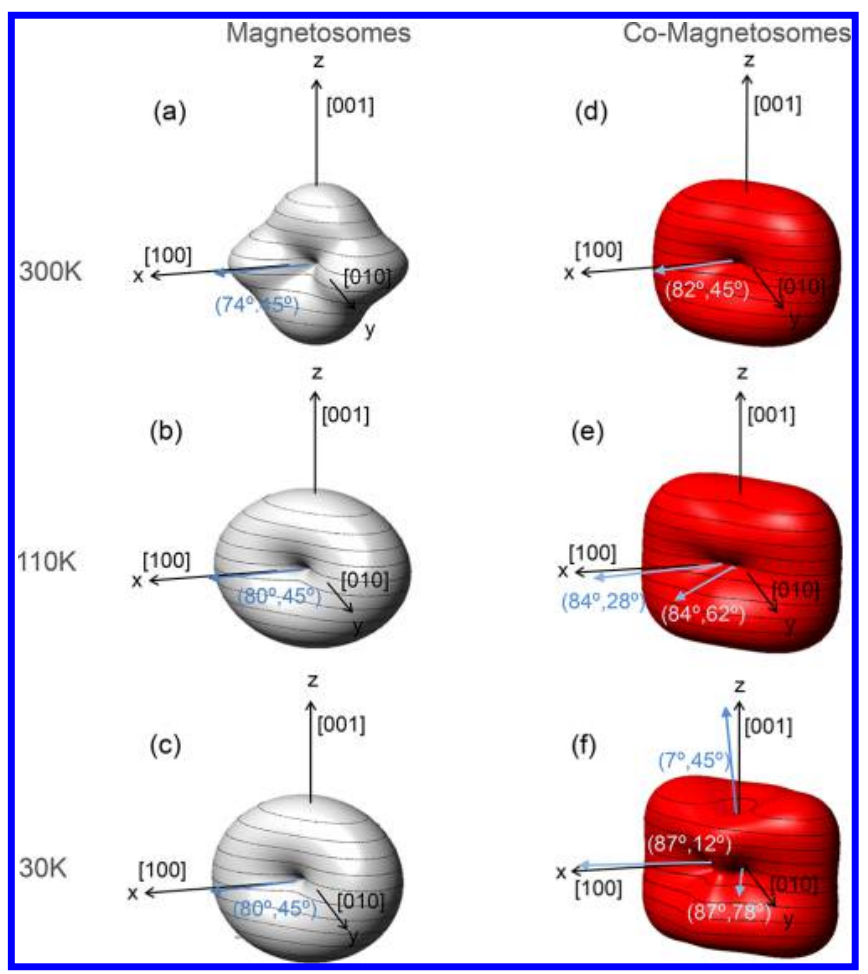

Figure 7. Zero-field energy landscapes at 300,110 , and $30 \mathrm{~K}$ of the control $(a-c)$ and Co-doped magnetosomes $(d-f)$ obtained from the simulations explained in the text. The blue arrows represent the effective easy axes, whose directions are given by $(\theta, \varphi)$, where $\theta$ is the polar angle and $\varphi$ is the azimuthal angle. A detailed view of the energy landscapes can be found in the Supporting Information (Movies S1S6).

contribution is to tilt slightly the direction of the uniaxial term set at $\left(\theta=80^{\circ}, \varphi=45^{\circ}\right)$.

At $110 \mathrm{~K}$ (Figure $7 \mathrm{~b}$ ), when $K_{\mathrm{c}} \approx 0$, the energy surface resembles a toroid that is flattened along the [001] direction. It is thus nearly a pure uniaxial anisotropy, and the corresponding effective easy axis is coincident with the uniaxial easy axis set for the uniaxial term in eq $2\left(\theta=80^{\circ}, \varphi=45^{\circ}\right)$. This term dominates the overall energy down to $30 \mathrm{~K}$ (Figure $7 \mathrm{c}$ ), where an almost perfect uniaxial symmetry is observed.

The effective uniaxial anisotropy observed in the control magnetosomes during the whole temperature range studied is in agreement with the observed reduced remanence values close to $\sim 0.5$ (Figure $5 \mathrm{e}$ ), as expected for randomly oriented uniaxial magnetic domains in the framework of the StonerWohlfarth model. $^{41}$

On the contrary, a different scenario is observed in the Codoped magnetosomes, as shown in Figure 7d-f (Movies S4S6). At $300 \mathrm{~K}$ the energy shows a single minimum, corresponding to an effective easy axis along the direction $(\theta$ $\left.=82^{\circ}, \varphi=45^{\circ}\right)$, only slightly tilted with respect to the uniaxial term set at $\left(\theta=80^{\circ}, \varphi=45^{\circ}\right)$. The cubic magnetocrystalline contribution to the energy is only reflected as a slight flattening of the energy surface at the [001] axis. As the temperature decreases, the ratio of $\left\langle K_{c}\right\rangle / K_{\mathrm{u}}$ increases, and as a consequence the contribution of the magnetocrystalline cubic anisotropy to the total energy acquires an increasingly more important role, and the system evolves from showing one $(300 \mathrm{~K})$ to two $(110$ $\mathrm{K})$ to finally three $(30 \mathrm{~K})$ effective easy axes. For $0 \leq\left\langle K_{\mathrm{c}}\right\rangle / K_{\mathrm{u}}$ $\leq 1$ there is a single easy axis, although with a nontoroidal symmetry. For $1<\left\langle K_{\mathrm{c}}\right\rangle / K_{\mathrm{u}} \leq 1.63$ there are two equivalent 
easy axes close to axes [100] and [010], and above 1.63 there are three easy axes, two of them equivalent (now even closer to [100] and [010]) and the third nearly coincident with [001]. Crossing points are located at $\sim 150 \mathrm{~K}$ (from uniaxial to biaxial) and at $100 \mathrm{~K}$ (from biaxial to triaxial). This is consistent with the reduced remanence increasing above 0.5 below $150 \mathrm{~K}$ because below this point the anisotropy is no longer uniaxial.

Finally, below $30 \mathrm{~K}$ the reduced remnant magnetization decreases remarkably from 0.65 at $30 \mathrm{~K}$ to near 0.5 at $5 \mathrm{~K}$, as observed in Figure 5e, and the hysteresis loops are no longer well reproduced following the same model, as sketched in Figure 6a, where uniaxial anisotropy has been assumed to keep an angle of $25^{\circ}$ with the $\langle 111\rangle$ toward $\langle 110\rangle$. Below $30 \mathrm{~K}$ the experimental loops can be accurately reproduced only if the uniaxial contribution undergoes a reorientation of $\sim 15^{\circ}$ toward directions [001] or [100] in the sketch of Figure 6a. This reorientation is accompanied by a slight decrease in the cubic anisotropy constant, as shown in Figure 6c. The underlying physical mechanism behind this effect remains unclear for us. A definite fact, however, is that the reorientation cannot be related to either shape anisotropy or to dipolar interaction effects but instead should be linked to the Co-doped magnetite phase itself. Additional insight provided by electronic transfer property measurements or more precise structural determination could shed light on this matter.

\section{CONCLUSIONS}

From the combination of structural and magnetic techniques we have observed that a low Co atomic percent (1\%), incorporated as $\mathrm{Co}^{2+}$ substituting $\mathrm{Fe}^{2+}$ into the magnetosome structure, adds a large positive contribution to the magnetocrystalline anisotropy of magnetosomes. This magnetocrystalline anisotropy is strongly dependent on temperature and competes with a uniaxial anisotropy that results from the shape anisotropy of the magnetosome and the dipolar interactions between them in the chain. On the basis of the slightly modified Stoner-Wohlfarth model and considering the different anisotropy contributions, we have been able to accurately reproduce the magnetic behavior of the control and Co-doped magnetosome chains and have determined the direction of the easy axes of the magnetization as a function of the temperature. In contrast with the control magnetosome chains, whose effective anisotropy is uniaxial in the whole temperature range, the effective anisotropy of Co-doped magnetosome chains changes appreciably with temperature, from uniaxial from 300 to $150 \mathrm{~K}$, to biaxial down to $100 \mathrm{~K}$ and triaxial below $100 \mathrm{~K}$. Further research effort should focus on the electronic state of the Co-doped magnetosomes at very low temperatures.

\section{ASSOCIATED CONTENT}

\section{(S Supporting Information}

The Supporting Information is available free of charge on the ACS Publications website at DOI: 10.1021/acs.jpcc.8b01187.

Movie S1. Video of the zero-field energy landscape at $300 \mathrm{~K}$ of the control magnetosomes obtained from the simulations explained in the text. (AVI)

Movie S2. Video of the zero-field energy landscape at $110 \mathrm{~K}$ of the control magnetosomes obtained from the simulations explained in the text. (AVI)
Movie S3. Video of the zero-field energy landscape at 30 $\mathrm{K}$ of the control magnetosomes obtained from the simulations explained in the text. (AVI)

Movies S4. Video of the zero-field energy landscape at $300 \mathrm{~K}$ of the Co-doped magnetosomes obtained from the simulations explained in the text. (AVI)

Movies S5. Video of the zero-field energy landscape at $110 \mathrm{~K}$ of the Co-doped magnetosomes obtained from the simulations explained in the text. (AVI)

Movies S6. Video of the zero-field energy landscape at 30 $\mathrm{K}$ of the Co-doped magnetosomes obtained from the simulations explained in the text. (AVI)

\section{AUTHOR INFORMATION}

\section{Corresponding Author}

*E-mail: malu.gubieda@ehu.eus.

ORCID $\odot$

Lourdes Marcano: 0000-0001-9397-6122

Aida Serrano: 0000-0002-6162-0014

Sergio Valencia: 0000-0002-3912-5797

M. Luisa Fdez-Gubieda: 0000-0001-6076-7738

Notes

The authors declare no competing financial interest.

\section{ACKNOWLEDGMENTS}

L.M. acknowledges the Basque Government for her fellowship (PRE_2015_1_0130). We acknowledge the technical and human support provided by SGIker (UPV/EHU). Funding from the Spanish Government (project nos. MAT2014-55049C2-R and MAT2017-83631-C3-R) and Basque Government (project n. IT711-13) is acknowledged. We thank the ESRF (CRG BM25 beamline-SpLine) and HZB for the allocation of synchrotron radiation beamtime and funding under the project CALIPSOplus (Grant Agreement 730872) from the EU Framework Programme for Research and Innovation HORIZON 2020. We thank R. Fernández-Pacheco for his assistance in the EDS measurements.

\section{REFERENCES}

(1) Blakemore, R. P. Magnetotactic Bacteria. Annu. Rev. Microbiol. 1982, 36, 217-238.

(2) Bazylinski, D. A.; Frankel, R. B. Magnetosome formation in prokaryotes. Nat. Rev. Microbiol. 2004, 2, 217-230.

(3) Alphandéry, E. Applications of Magnetosomes Synthesized by Magnetotactic Bacteria in Medicine. Front. Bioen. Biotechnol. 2014, 2, 5 .

(4) Mériaux, S.; Boucher, M.; Marty, B.; Lalatonne, Y.; Prévéral, S.; Motte, L.; Lefevvre, C. T.; Geffroy, F.; Lethimonnier, F.; Péan, M.; et al. Magnetosomes, Biogenic Magnetic Nanomaterials for Brain Molecular Imaging with 17.2 T MRI Scanner. Adv. Healthcare Mater. 2015, 4, 1076-1083.

(5) Sun, J.; Tang, T.; Duan, J.; Xu, P.-X.; Wang, Z.; Zhang, Y.; Wu, L.; Li, Y. Biocompatibility of bacterial magnetosomes: Acute toxicity, immunotoxicity and cytotoxicity. Nanotoxicology 2010, 4, 271-283.

(6) Muela, A.; Muñoz, D.; Martín-Rodríguez, R.; Orue, I.; Garaio, E.; Abad Díaz de Cerio, A.; Alonso, J.; García, J. Á.; Fdez-Gubieda, M. L. Optimal Parameters for Hyperthermia Treatment Using Biomineralized Magnetite Nanoparticles: Theoretical and Experimental Approach. I. Phys. Chem. C 2016, 120, 24437-24448.

(7) Orlando, T.; Mannucci, S.; Fantechi, E.; Conti, G.; Tambalo, S.; Busato, A.; Innocenti, C.; Ghin, L.; Bassi, R.; Arosio, P.; et al. Characterization of magnetic nanoparticles from Magnetospirillum gryphiswaldense as potential theranostics tools: Characterization of 
Magnetic Nanoparticles from Magnetotactic Bacteria. Contrast Media Mol. Imaging 2016, 11, 139-145.

(8) Mannucci, S.; Ghin, L.; Conti, G.; Tambalo, S.; Lascialfari, A.; Orlando, T.; Benati, D.; Bernardi, P.; Betterle, N.; Bassi, R.; et al. Magnetic Nanoparticles from Magnetospirillum gryphiswaldense Increase the Efficacy of Thermotherapy in a Model of Colon Carcinoma. PLoS One 2014, 9, e108959.

(9) Staniland, S.; Williams, W.; Telling, N.; van der Laan, G.; Harrison, A.; Ward, B. Controlled cobalt doping of magnetosomes in vivo. Nat. Nanotechnol. 2008, 3, 158-162.

(10) Tanaka, M.; Brown, R.; Hondow, N.; Arakaki, A.; Matsunaga, T.; Staniland, S. Highest levels of $\mathrm{Cu}, \mathrm{Mn}$ and $\mathrm{Co}$ doped into nanomagnetic magnetosomes through optimized biomineralisation. $L$. Mater. Chem. 2012, 22, 11919.

(11) Prozorov, T.; Perez-Gonzalez, T.; Valverde-Tercedor, C.; Jimenez-Lopez, C.; Yebra-Rodriguez, A.; Körnig, A.; Faivre, D.; Mallapragada, S. K.; Howse, P. A.; Bazylinski, D. A.; et al. Manganese incorporation into the magnetosome magnetite: magnetic signature of doping. Eur. J. Mineral. 2014, 26, 457-471.

(12) Li, J.; Menguy, N.; Arrio, M.-A.; Sainctavit, P.; Juhin, A.; Wang, Y.; Chen, H.; Bunau, O.; Otero, E.; Ohresser, P.; et al. Controlled cobalt doping in the spinel structure of magnetosome magnetite: new evidences from element- and site-specific X-ray magnetic circular dichroism analyses. I. R. Soc., Interface 2016, 13, 20160355.

(13) Alphandéry, E.; Carvallo, C.; Menguy, N.; Chebbi, I. Chains of Cobalt Doped Magnetosomes Extracted from AMB-1 Magnetotactic Bacteria for Application in Alternative Magnetic Field Cancer Therapy. I. Phvs. Chem. C 2011, 115, 11920-11924.

(14) Amemiya, Y.; Arakaki, A.; Staniland, S. S.; Tanaka, T.; Matsunaga, T. Controlled formation of magnetite crystal by partial oxidation of ferrous hydroxide in the presence of recombinant magnetotactic bacterial protein Mms6. Biomaterials 2007, 28, 53815389.

(15) Lang, C.; Schüler, D.; Faivre, D. Synthesis of Magnetite Nanoparticles for Bio- and Nanotechnology: Genetic Engineering and Biomimetics of Bacterial Magnetosomes. Macromol. Biosci. 2007, 7, $144-151$.

(16) Arakaki, A.; Masuda, F.; Amemiya, Y.; Tanaka, T.; Matsunaga, T. Control of the morphology and size of magnetite particles with peptides mimicking the Mms6 protein from magnetotactic bacteria. $I$. Colloid Interface Sci. 2010, 343, 65-70.

(17) Galloway, J. M.; Arakaki, A.; Masuda, F.; Tanaka, T.; Matsunaga, T.; Staniland, S. S. Magnetic bacterial protein Mms6 controls morphology, crystallinity and magnetism of cobalt-doped magnetite

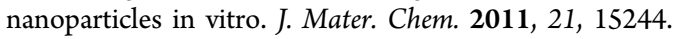

(18) Larumbe, S.; Gómez-Polo, C.; Pérez-Landazábal, J. I.; GarcíaPrieto, A.; Alonso, J.; Fdez-Gubieda, M. L.; Cordero, D.; Gòmez, J. Ni Doped $\mathrm{Fe}_{3} \mathrm{O}_{4}$ Magnetic Nanoparticles. I. Nanosci. Nanotechnol. 2012, $12,2652-2660$.

(19) Bazylinski, D. A.; Garrattreed, A. J.; Abedi, A.; Frankel, R. B. Copper Association with Iron Sulfide Magnetosomes in a Magnetotactic Bacterium. Arch. Microbiol. 1993, 160, 35-42.

(20) Li, R. A study of the magnetotactic bacteria and their magnetosomes from a section of loess in the Shanxi Province. Diaiu Huaxue 1996, 25, 251-254.

(21) Lee, S. W.; Bae, S.; Takemura, Y.; Shim, I. B.; Kim, T. M.; Kim, J.; Lee, H. J.; Zurn, S.; Kim, C. S. Self-heating characteristics of cobalt ferrite nanoparticles for hyperthermia application. I. Magn. Magn. Mater. 2007, 310, 2868-2870.

(22) Joshi, H. M.; Lin, Y. P.; Aslam, M.; Prasad, P. V.; Schultz-Sikma, E. A.; Edelman, R.; Meade, T.; Dravid, V. P. Effects of Shape and Size of Cobalt Ferrite Nanostructures on Their MRI Contrast and Thermal Activation. I. Phvs. Chem. C 2009, 113, 17761-17767.

(23) Heyen, U.; Schüler, D. Growth and magnetosome formation by microaerophilic Magnetospirillum strains in an oxygen-controlled fermentor. Appl. Microbiol. Biotechnol. 2003, 61, 536-544.

(24) Grünberg, K.; Wawer, C.; Tebo, B. M.; Schüler, D. A Large Gene Cluster Encoding Several Magnetosome Proteins Is Conserved in Different Species of Magnetotactic Bacteria. Appl. Environ. Microbiol. 2001, 67, 4573-4582.

(25) Schneider, C. A.; Rasband, W. S.; Eliceiri, K. W. NIH Image to ImageJ: 25 years of image analysis. Nat. Methods 2012, 9, 671-675.

(26) Castro, G. R. Optical design of the general-purpose Spanish Xray beamline for absorption and diffraction. L. Sunchrotron Radiat. 1998, 5, 657-660.

(27) Ravel, B.; Newville, M. ATHENA, ARTEMIS, HEPHAESTUS: data analysis for X-ray absorption spectroscopy using IFEFFIT. I. Sunchrotron Radiat. 2005, 12, 537-541.

(28) Grabis, J.; Nefedov, A.; Zabel, H. Diffractometer for soft x-ray resonant magnetic scattering. Rev. Sci. Instrum. 2003, 74, 4048-4051.

(29) Abrudan, R.; Brüssing, F.; Salikhov, R.; Meermann, J.; Radu, I.; Ryll, H.; Radu, F.; Zabel, H. ALICE-An advanced reflectometer for static and dynamic experiments in magnetism at synchrotron radiation facilities. Rev. Sci. Instrum. 2015, 86, 063902.

(30) Fdez-Gubieda, M. L.; Muela, A.; Alonso, J.; García-Prieto, A.; Olivi, L.; Fernandez-Pacheco, R.; Barandiaran, J. M. Magnetite Biomineralization in Magnetospirillum gryphiswaldense: Time-Resolved Magnetic and Structural Studies. ACS Nano 2013, 7, 3297-3305.

(31) Carta, D.; Casula, M. F.; Falqui, A.; Loche, D.; Mountjoy, G.; Sangregorio, C.; Corrias, A. A Structural and Magnetic Investigation of the Inversion Degree in Ferrite Nanocrystals $\mathrm{MFe}_{2} \mathrm{O}_{4}(\mathrm{M}=\mathrm{Mn}, \mathrm{Co}$, Ni). I. Phvs. Chem. C 2009, 113, 8606-8615.

(32) Wilke, M.; Farges, F.; Petit, P.-E.; Brown, G. E., Jr.; Martin, F. Oxidation state and coordination of $\mathrm{Fe}$ in minerals: An Fe K-XANES spectroscopy study. Am. Mineral. 2001, 86, 714-730.

(33) van der Laan, G.; Figueroa, A. I. Coord. Chem. Rev. 2014, 277, 95-129.

(34) Pattrick, R. A. D.; van der Laan, G.; Henderson, C. M. B.; Kuiper, P.; Dudzik, E.; Vaughan, D. J. Cation site occupancy in spinel ferrites studied by X-ray magnetic circular dichroism: developing a method for mineralogists. Eur. I. Mineral. 2002, 14, 1095-1102.

(35) Prozorov, R.; Prozorov, T.; Mallapragada, S. K.; Narasimhan, B.; Williams, T. J.; Bazylinski, D. A. Magnetic irreversibility and the Verwey transition in nanocrystalline bacterial magnetite. Phvs. Rev. B: Condens. Matter Mater. Phvs. 2007, 76, 054406.

(36) Marcano, L.; García-Prieto, A.; Muñoz, D.; Fernández Barquín, L.; Orue, I.; Alonso, J.; Muela, A.; Fdez-Gubieda, M. Influence of the bacterial growth phase on the magnetic properties of magnetosomes synthesized by Magnetospirillum gryphiswaldense. Biochim. Biophvs. Acta. Gen. Subi. 2017, 1861, 1507-1514.

(37) Fock, J.; Bogart, L. K.; González-Alonso, D.; Espeso, J. I.; Hansen, M. F.; Varón, M.; Frandsen, C.; Pankhurst, Q. A. On the 'centre of gravity' method for measuring the composition of magnetite/maghemite mixtures, or the stoichiometry of magnetitemaghemite solid solutions, via ${ }^{57} \mathrm{Fe}$ Mössbauer spectroscopy. $[$. Phvs. D: Appl. Phvs. 2017, 50, 265005.

(38) Fischer, H.; Mastrogiacomo, G.; Loffler, J. F.; Warthmann, R. J.; Weidler, P. G.; Gehring, A. U. Ferromagnetic resonance and magnetic characteristics of intact magnetosome chains in Magnetospirillum gryphiswaldense. Earth Planet. Sci. Lett. 2008, 270, 200-208.

(39) Kronmüller, H.; Walz, F. Magnetic after-effects in $\mathrm{Fe}_{3} \mathrm{O}_{4}$ and vacancy-doped magnetite. Philos. Mag. B 1980, 42, 433-452.

(40) Muxworthy, A. R; McClelland, E. Review of the lowtemperature magnetic properties of magnetite from a rock magnetic perspective. Geophvs. I. Int. 2000, 140, 101-114.

(41) Stoner, E.; Wohlfarth, E. A mechanism of magnetic hysteresis in heterogeneous alloys. Philos. Trans. R. Soc., A 1948, 240, 599-642.

(42) Geoghegan, L. J.; Coffey, W. T.; Mulligan, B. Differential Recurrence Relations for Non-Axially Symmetric Rotational FokkerPlanck Equations. Adv. Chem. Phys. 2007, 100, 475-641.

(43) Carrey, J.; Mehdaoui, B.; Respaud, M. Simple models for dynamic hysteresis loop calculations of magnetic single-domain nanoparticles: Application to magnetic hyperthermia optimization. $I$. Appl. Phvs. 2011, 109, 083921.

(44) Huizar-Felix, A. M.; Munoz, D.; Orue, I.; Magen, C.; Ibarra, A.; Barandiaran, J. M.; Muela, A.; Fdez-Gubieda, M. L. Assemblies of 
magnetite nanoparticles extracted from magnetotactic bacteria: A magnetic study. Appl. Phvs. Lett. 2016, 108, 063109.

(45) Charilaou, M.; Kind, J.; García-Rubio, I.; Schüler, D.; Gehring, A. U. Magnetic anisotropy of non-interacting collinear nanocrystalchains. Appl. Phvs. Lett. 2014, 104, 112406.

(46) Charilaou, M.; Winklhofer, M.; Gehring, A. U. Simulation of ferromagnetic resonance spectra of linear chains of magnetite nanocrystals. I. Appl. Phys. 2011, 109, 093903.

(47) Gehring, A. U.; Fischer, H.; Charilaou, M.; García-Rubio, I. Magnetic anisotropy and Verwey transition of magnetosome chains in Magnetospirillum gryphiswaldense: Magnetic anisotropy and Verwey transition. Geophvs. I. Int. 2011, 187, 1215-1221.

(48) Orue, I.; Marcano, L.; Bender, P.; García-Prieto, A.; Valencia, S.; Mawass, M. A.; Gil Carton, D.; Alba Venero, D.; Honecker, D.; GarcíaArribas, A.; et al. Configuration of the magnetosome chain: a natural magnetic nanoarchitecture. Nanoscale 2018, DOI: 10.1039/ C7NR08493E.

(49) Mann, S.; Frankel, R. B.; Blakemore, R. P. Structure, morphology and crystal growth of bacterial magnetite. Nature 1984, 310, 405.

(50) Körnig, A.; Winklhofer, M.; Baumgartner, J.; Gonzalez, T. P.; Fratzl, P.; Faivre, D. Magnetite Crystal Orientation in Magnetosome Chains. Adv. Funct. Mater. 2014, 24, 3926-3932.

(51) Thomas, J. M.; Simpson, E. T.; Kasama, T.; Dunin-Borkowski, R. E. Electron Holography for the Study of Magnetic Nanomaterials. Acc. Chem. Res. 2008, 41, 665-674.

(52) Bickford, L. R; Brownlow, J. M.; Penoyer, R. F. Magnetocrystalline anisotropy in cobalt-substituted magnetite single crystals. Proc. IEEE 1957, 104, 238-244.

(53) Abe, K.; Miyamoto, Y.; Chikazumi, S. Magnetocrystalline Anisotropy of Low Temperature Phase of Magnetite. I. Phys. Soc. Ipn. 1976, 41, 1894.

(54) Tachiki, M. Origin of the Magnetic Anisotropy Energy of Cobalt Ferrite. Prog. Theor. Phvs. 1960, 23, 1055-1072.

(55) Zhang, Q.; Castellanos-Rubio, I.; Munshi, R.; Orue, I.; Pelaz, B.; Gries, K. I.; Parak, W. J.; del Pino, P.; Pralle, A. Model Driven Optimization of Magnetic Anisotropy of Exchange-Coupled CoreShell Ferrite Nanoparticles for Maximal Hysteretic Loss. Chem. Mater. 2015, 27, 7380-7387. 